\title{
Prevalence of Intestinal Parasites and Associated Risk Factors at Red Cross Clinic and Chelaleki Health Center, East Wollega Zone, Ethiopia
}

\author{
Addis Adera Gebru ${ }^{1}$, Birhan Alemnew Tamene ${ }^{1}$, Asmamaw Demis Bizuneh ${ }^{1}$, Yonas Yimam Ayene ${ }^{1}$, \\ Zemenu Mengistie Semene ${ }^{1}$, Ambachew Woreta Hailu ${ }^{2}$, Tefera Nigussie ${ }^{3}$, , Abdi Samuel ${ }^{4}$, \\ Markos Kidane Assefa ${ }^{5}$ \\ ${ }^{1}$ Department of Nursing, Faculty of Health Sciences, Woldia University, North Wollo, Amhara Region, Ethiopia \\ ${ }^{2}$ Department of Biology, Faculty of Natural and Computational Sciences, Woldia University, North Wollo, Amhara Region, Ethiopia \\ ${ }^{3}$ Department of Nursing, Faculty of Health Sciences, Debre Birhan University, Debre Birhan, Ethiopia \\ ${ }^{4}$ Department of Medical Laboratory Sciences, College of Medical and Health Sciences, Wollega University, Nekemte, Ethiopia \\ ${ }^{5}$ Huwawei Company, Addis Ababa Woreda Net project, Addis Ababa, Ethiopia
}

\section{Email address:}

addisaderagebru@gmail.com (A. A. Gebru), birhanalemnew12@gmail.com (B. A. Bizuneh), yonasyimamayene@gmail.com (Y. Y. Ayene), markos.kidane@yahoo.com (M. K. Assefa),zemenumengistie@yahoo.com (Z. M. Semene), ambalake@gmail.com (A. W. Hailu), addisaderagebru@gmail.com (T. Nigussie)

\section{To cite this article:}

Addis Adera Gebru, Birhan Alemnew Tamene, Asmamaw Demis Bizuneh, Yonas Yimam Ayene, Zemenu Mengistie Semene, Ambachew Woreta Hailu, Tefera Nigussie, Abdi Samuel, Markos Kidane Assefa. Prevalence of Intestinal Parasites and Associated Risk Factors at Red Cross Clinic and Chelaleki Health Center, East Wollega Zone, Ethiopia. Science Journal of Public Health. Vol. 3, No. 4, 2015 , pp. 445-452. doi: $10.11648 /$ j.sjph.20150304.11

\begin{abstract}
Introduction: Intestinal parasites present throughout the world in various degree of prevalence are the major health problems in areas where there is overcrowding, poor environmental sanitation, and personal hygienic practice especially in developing countries like Ethiopia. They are known to cause major morbidity and mortality rate in these countries. This study will provide valuable information about the prevalence of intestinal parasites and help in designing effective preventive and control strategies in the study area. Objective: The aim of this study was to determine the prevalence of intestinal parasites and the associated risk factors among patients requested for stool examination at Red Cross Clinic and Chelaleki Health Center, East Wollega Zone, Ethiopia. Method: A facility-based cross sectional study was conducted from April 02-23, 2014 at Red Cross Clinic and Chelaleki Health Center. 368 study subjects were included in the study using convenience sampling technique and the socio-demographic data of the study subjects were collected using semi-structured questionnaire. Stool specimens were examined microscopically and data was analyzed using SPSS version 16.Result: A total of 368 stool specimens were collected of which 139(37.8\%) respondents were affected with intestinal parasites and 229(62.2\%) were not found any intestinal parasitic infection. The two most prevalent intestinal parasites in this study were E. histolytica (16.3\%) and G. lamblia (9\%). From the total positive cases, 50(50\%) were in age group $\leq 14$ years, 64(37\%) in 15-29 years, $12(26.6 \%)$ in $\geq 45$ years and $13(26 \%)$ in 30-44 years. Conclusion: In this study, children were highly affected than the other age groups and infections were caused by poor environmental and personal hygiene.
\end{abstract}

Keywords: Intestinal Parasite, Prevalence, Cross-Sectional, Red Cross Clinic, Chelaleki Health Center, Ethiopia

\section{Introduction}

Intestinal parasites (IPs) are organisms living in the body of other organism having some metabolic dependence (1). Generally, IPs (protozoan or helminthes) are more common in tropics and subtropics than elsewhere in the world (2). Current estimates showed that at least more than one quarter of the world's population is chronically infected with IP and that most of these infected people live in developing countries $(3,4)$. The prevalence and profile of intestinal parasitic infections vary widely among continents and within countries sub regions. In South Africa, school children in the district of 
Vhembe, the parasite Entamoeba histolytica/dispar (E.histolytica/dispar) (10\%) and Giardia lamblia (G.lambia) (12\%) are the leading cause of diarrhea in children 5 to 15 years (5) while in the city of cape, the main infectious is caused by A.lumbricoides (24.8\%), T.trichuria (50.0\%), G.lamblia (17.3\%) and Hymenolepis nana (H.nana) (2.2\%) (6). In India, Karnataba city, A.lumbricoides was the predominant single species in both Urban (56\%) and rural population (79.2\%) (7). In Latin America, school children in the Palajunoj valley, Guatemala, the prevalence of the parasite was A.lumbricoides(17.7\%), E. histolytica/dispar (16.1\%), G.lambia(10.9\%), H.nana (5.4\%) and Blastocytishominis $(2.8 \%)(8)$. Few studies have reported the world wide. For instance, In Saudi Arabia, a cross sectional study conducted on the prevalence of IPs among patients of AlNoor specialist Hospital, Makkah indicated that the overall prevalence of IPs was $6.2 \%(740 / 12,054)$. Majority of patients were infected by E. histolytica/dispar (4.7\%) followed by Giardia lamblia (1.3\%), while Ankylostoma duodenale $(0.02 \%)$ exhibited the minimum prevalence. Parasitic infections were more frequent in non-Saudis than Saudi patients $(7.1 \%$ vs. $5.8 \%)$. There was no significant difference between males and females regarding parasitic infections, with a female to male ratio of 1:1.08. There was a higher prevalence in patients under 5 years of age $(9.1 \%)$ followed by patients aged $5-14$ years $(7.5 \%)(9)$. In Thailand, a study was conducted on the prevalence of IPs among food handlers in Sai-Yok District, Kanchanburi province and indicated that the prevalence of intestinal parasites was $10.3 \%(28 / 273)$. Among these, hookworm was the most common $(70.0 \%)$, followed by $\mathrm{T}$. trichuria $(10.0 \%)$, Blastocystis hominis (3.3\%), and Giardia lamblia $(3.3 \%)$. In many of the developing countries including Ethiopia, the most prevalent and important helminthes are those of the soil transmitted nematodes. Chronic gut infection in humans commonly results from nematodes, particularly that of Ascaris lumbricoides, Trichuris trichuria and Hookworm. For instance, it was found that helminthiasis is the second most common cause of outpatient morbidity next to malaria in Ethiopia $(10,11)$ where children are the most affected group and serve both as source of infection and as victims, thus contributing to transmission of most parasitic infections within the country (12).Ethiopia has one of the lowest quality drinking water supply and latrine coverage in the world. As comparison by 2000 , Ethiopia had only $12 \%$ latrine coverage, while Kenya had $87 \%(13)$. Many reports illustrated that A. lumbricoides is the most prevalent intestinal parasite in different community, usually occurring together with Trichuris infections (14). Hookworm infection, Strongylodiasis and Enterobiasis are also public health problems though the magnitude is lesser compared to Ascariasis. The prevalence of Taeniasis ranges from $1-48 \%$ and the infection rate with H.nana is 3-61 \% (15). The highest prevalence of S. Mansoni was reported in the age group of $10-14$ years $(17.8 \%)$ followed by the age group 15 and above $11.6 \%$ and the difference was statistically significant( 16 ). In addition, It is estimated that about $10 \%$ of the population of developing countries including Ethiopia is infected with IP and that such cases can be adequately controlled through improvements in hygiene, potable water and sewage treatment (17). Integrated control programs including periodic deworming, construction of public toilets, creating awareness regarding the importance of washing hands after defecation and the impact of swimming in contaminated water are needed to reduce intestinal parasitic disease transmission (18). Several factors like climatic conditions, poor sanitation, unsafe drinking water and lack of toilet facilities are the many contributors for the high prevalence of IPs in the tropical and sub-tropical countries (19).In addition, Intestinal parasitic agents increase in polluted environments such as refuse heaps, gutters and sewage units in and around human dwelling and living conditions of people in crowded or unhealthy situations(20). Besides factors such as socio-economic status, poor sanitation, inadequate medical care, and absence of safe drinking water supplies, it has also been reported that unsanitary sewage disposal, the habit of eating raw or semi-raw fish and the practice of allowing untreated infected sewage to drain in fresh water lakes are responsible for the establishment and maintenance of the parasites (21). Environmental factors also play a role in the incidence of intestinal parasitic infection as hot and humid tropical climate favor increased parasite prevalence (22). Based on variation in climatic and geographical zones in Ethiopia, it should be evident that there are macro and micro environmental factors contributing to the differences in prevalence of IPs (23).From preliminary observation it was found that no study has so far been conducted on IPs in Nekemte town. Therefore, the aim of this study is to determine the prevalence of IPs and associated risk factors at Red Cross Clinic and Chelaleki Health Center.

\section{Methods and Materials}

\subsection{Study Area and Study Period}

This study was conducted in Red Cross Clinic and Chelaleki Health Center, East Wollega, Ethiopia. Red Cross Clinic and Chelaleki Health Center are located in Nekemte Town, a capital city of East Wollega Zone. Nekemte Town is found at a distance of 331 kilometers from Addis Ababa in the West direction. Geographically, it is located at N90 S12611and E3603214811, latitude and longitude, respectively. The town is found at altitude of 2,118 meter above sea level. It has a climatic condition of most of the area is "Woyina Dega" and characterized by warm climate with mean annual maximum temperature of $280 \mathrm{c}$ and minimum of 170c, respectively. According to the 2012 census, Nekemte town has a total population of about 110,688. Red Cross Clinic and Chelaleki Health Center are 
some of the health service providing institutions in the town. The study was conducted from April 02-23, 2014 in the Health Center.

\subsection{Study Design}

Facility based cross sectional study design was employed.

\subsection{Study Population}

Sampled an individual who had visited Red Cross Clinic and Chelaleki Health Center, East Wollega Zone, Ethiopia during the study period and their age ranges from 14-45 years. This study including all consented patients requested for stool examination and who were not taking treatment for intestinal parasitic infections. However, all patients requested for stool examination those who were not consented and who were taking treatment for intestinal parasitic infections were excluded from this study.

\subsection{The Sample Size}

The sample size was determined by using a Population formula considering the following assumptions: We take the prevalence rate of IPs from study conducted at Fenan Medical Center East Wollega which is $64.9 \%(38)$. Total sample sizes were 368 .

\subsection{Sampling Technique}

A sampling technique used in this study was convenience sampling technique which is used to include all consented patients requested for stool examination during the study period.

\subsection{Instruments and Measurements}

Pretested and a semi-structured questionnaire was administered to generate information on socio demographic characteristics, pattern of latrine usage, sources of drinking water and personal hygiene.

\subsection{Data Collection Procedure}

The data were collected for 368 stool specimens in study area. The patients were interviewed face to face by principal investigator. Each study subject was provided with stool cup and applicator stick and informed to bring small portion of stool sufficient enough for direct saline wet mount technique. Finally, each sample was examined using microscope by principal investigator and confirmed by senior laboratory technologist from Chelaleki Health Center and Red Cross Clinic.

\subsection{Data Processing and Analysis}

The questionnaire checked for completeness and consistency and entered into SPSS statistical package version 16. Frequencies, proportion and summary statistically was used to describe variables. The data collected from semistructured questionnaire and stool examination was filled appropriately in dummy tables and the overall prevalence of IPs and each species was calculated using scientific calculator. The result was represented using tables and association of IPs with independent variables was determined using the chi-square and P-value less than 0.05 was considered as level of significance.

\subsection{Data Quality Assurance}

The following measures were taken to control the quality of data.

- Specimens were collected, processed and examined by following standard operational procedure (SOP).

- Materials and equipment's were checked for proper functioning.

- Stool examination results were reported after confirmed by senior laboratory technologist.

- Every collected sample test results were registered in the appropriate format.

Finally, the data was analyzed and interpreted accordingly

\subsection{Data Quality Control}

To ensure the quality of data, first the questionnaire was pretested. The pretested was conducted in $5 \%$ of the participants at randomly selected districts away from the study districts. Training was given for the data collectors and supervisors before the actual data collection. Every day after data collection, questionnaires were reviewed and checked for completeness, accuracy and clarity by the supervisors and principal investigator.

\subsection{Ethical Consideration}

The official permission letter for ethical clearance was obtained from Wollega University Research Ethics Committee through the department of Medical Laboratory Science. Further permission was obtained from Red Cross Clinic and Chelaleki Health Center and Laboratory staffs. The respondents were informed about the objective and purpose of the study and verbal consent was obtained from each respondent before starting the interview. The information obtained at each course of study was kept confidential.

\section{Result}

\subsection{Socio-Demographic Characteristics of the Study Participants}

A total of 368 subjects were participated in this study, among them, 174(47.3\%) were males and 194(52.7\%) were females. One hundred(27.2\%), 173(47\%), 50(13.6\%) and $45(12.2 \%)$ study participants were in age group $\leq 14$ years, 15-29 years, 30-44 years and $\geq 45$ years, respectively. Regarding religion, 196(53.3\%) were Protestants, 136(37\%) were Orthodox, 21(5.7\%) were Muslims, 13(3.5\%) were Catholic and $2(0.5 \%)$ were others. Two hundred twenty five $(61.1 \%)$ and $143(38.9 \%)$ of the study subjects were urban and rural residents, respectively. About 167(45.9\%), $63(17.1 \%), 48(13 \%), 36(9.8 \%), 33(9 \%)$ and $21(5.2 \%)$ of the study participants were students, farmers, housewives, 
merchants, governmental employees and others, respectively. From the total of 368 respondents, 34(9.2\%), $101(27.4 \%), 29(7.9 \%), 31(8.5 \%)$ respondents had monthly income of $<300$ birr, 300-900 birr, 1000-1500 birr and $>1500$ birr, respectively. About $173(47 \%)$ of the study participants were students, children and handicaps. Furthermore, 221(60\%), 79(21.5\%), 67(18.2\%) and 1(0.3\%) of the study participants get water from pipe, spring, well and stream, respectively.

Table 1. Socio-demographic characteristics of study participants at Red Cross Clinic and Chelaleki Health Center, East Wollega Zone, Ethiopia ,April, 2014.

\begin{tabular}{lll}
\hline Characteristics & Frequency $\mathbf{N}=\mathbf{3 6 8}$ & Percentage (\%) \\
\hline Age & 100 & $27.2 \%$ \\
$>14$ years & 173 & $47 \%$ \\
15-19 years & 50 & $136 \%$ \\
30-44 years & 50 & $136 \%$ \\
$<45$ years & 45 & $12.2 \%$ \\
Sex & & \\
Male & 174 & $47.3 \%$ \\
female & 194 & $52.7 \%$ \\
Religion & & \\
Protestant & 196 & $53.3 \%$ \\
Orthodox & 136 & $37 \%$ \\
Muslim & 21 & $5.7 \%$ \\
Catholic & 2 & $0.5 \%$ \\
Residence & & \\
Urban & 225 & $61 \%$ \\
rural & 143 & $38.9 \%$ \\
Occupation & & \\
Students & 167 & $45.9 \%$ \\
Farmer & 63 & $17.1 \%$ \\
House wives & 48 & $13 \%$ \\
Merchant & 36 & 9.8 \\
\hline
\end{tabular}

\begin{tabular}{lll}
\hline Characteristics & Frequency $\mathbf{N = 3 6 8}$ & Percentage (\%) \\
\hline $\begin{array}{l}\text { Government employee } \\
\text { Others }\end{array}$ & 33 & $9 \%$ \\
Monthly income & 21 & $5.2 \%$ \\
$<300$ birr & & \\
$300-900$ birr & 34 & $9.2 \%$ \\
$1000-1500$ & 101 & $27.4 \%$ \\
$<1500$ & 29 & $7.9 \%$ \\
Water source & 31 & $8.5 \%$ \\
Pipe & & \\
Spring & 221 & $60 \%$ \\
Well & 79 & $21 \%$ \\
stream & 67 & $18.2 \%$ \\
\hline & 1 & $0.3 \%$ \\
\hline
\end{tabular}

\subsection{Prevalence of Intestinal Parasitic Infection (IPI)}

The overall prevalence IPI in Chelaleki Health Center and Red Cross Clinic was 37.8 \%( 139/368). E. histolytica/dispar $60(16.3 \%)$ was the most predominant parasite followed by $\mathrm{G}$. lamblia 33(9\%), A. lumbricoides 19(5.2\%), Hookworm $14(3.8 \%)$ and H.nanal $(0.3 \%)$. From the total infected cases, about $91.4 \%(127 / 139), 7.2 \%(10 / 139)$ and $1.4 \%(2 / 139)$ were single, double and triple infections, respectively. There were $6(2.9 \%), 2(0.9 \%), 2(0.9 \%)$ and $1(0.5 \%)$ (E. histolytica and G.lamblia), (A.lumbricoides and E.histolytica), (E.histolytica and Hookworm) and (G.lamblia and H.nana) double infections. Furthermore, there were two different triple infections with A. lumbricoides/ E.histolytica/ Hookworm (0.5\%) and E.histolytica / G.lamblia/ Hookworm $(0.5 \%)$.In this study, children were highly affected than the other age groups and infections were caused by poor environmental and personal hygiene.

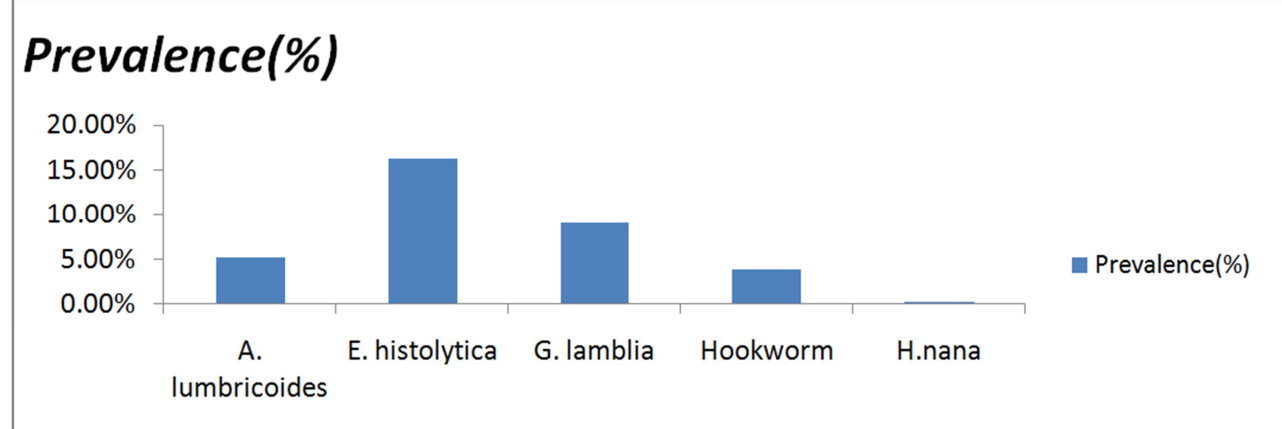

Types of Parasites

Figure 1. Prevalence of single parasitic infection in the study participants at Red Cross Clinic and Chelaleki Heath Center from April, 2014.

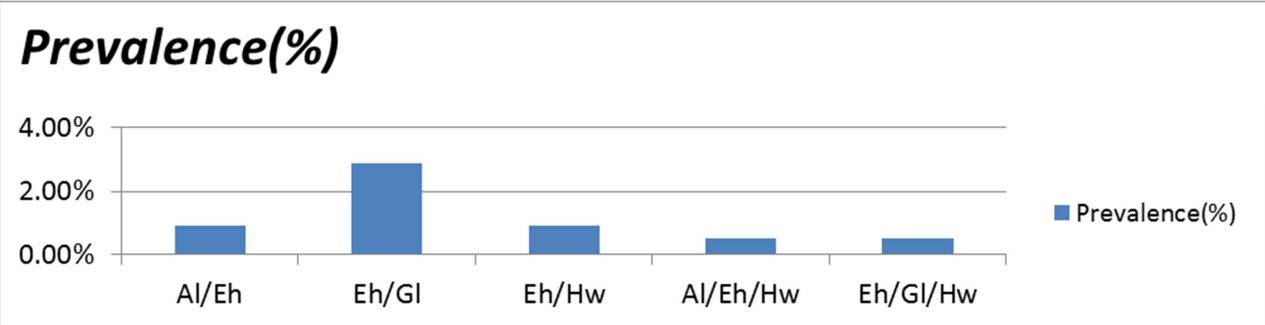

Types of parasites: $\mathrm{Al}=\mathrm{A}$.lumbricoides; $\mathrm{Eh}=\mathrm{E}$. histolytica; $\mathrm{Gl}=\mathrm{G} . \mathrm{lamblia} ; \mathrm{Hn}=\mathrm{H} . n a n a ; \mathrm{Hw}=\mathrm{Hookworm}$

Figure 2. Prevalence of multiple parasitic infections in the study participants at Red Cross Clinic and Chelaleki Heath Center April, 2014. 
Of the total study subjects, about 225(61.1\%) and $143(38.9 \%)$ study subjects were urban and rural residents, respectively. IPI was more prevalent in rural study participants $88(61.5 \%)$ than urban study participants $51(27.7 \%)$. From the total positive cases, 50 (50\%) were in age group $\leq 14$ years, $64(37 \%)$ in $15-29$ years, $12(26.6 \%)$ in $\geq 45$ years and $13(26 \%)$ in $30-44$ years. In this study, children were highly affected than the other age groups and infections were caused by poor environmental and personal hygiene. Residence and IPI showed a statistically significant association $(\mathrm{P}=0.01)$.From the total study participants, IPI was more prevalent in Orthodox 46.3\% (63/136) and Catholic $46.2 \%(6 / 13)$ religion followers. However, there was no significant association between religion and IPI $(\mathrm{P}=0.061)$. Of the total study participants, 221(60\%), $67(18.2 \%), 79(21.5 \%)$, and $1(0.3 \%)$ of study participants get water from pipe, well, spring and stream, respectively. Intestinal parasitic infection was more prevalent among study subjects whose source of water supply was well $65.7 \%$ (44/67) and spring 50.6\% (40/79). Intestinal parasitic infection was lower among study subjects who get water from pipe. Source of water supply and IPI was statistically associated with IPI $(\mathrm{P}=0.002)$. About $45.9 \%(167 / 368)$, $17.1 \%(63 / 368), 13 \%$ (48/368), $9.8 \%(36 / 368), 9 \%(33 / 368)$ and $5.2 \%(21 / 368)$ study subjects were students, farmers, house wives, merchants, governmental employees and others, respectively. There was higher prevalence of IPs among farmers $(60.3 \%)$ and students $(41.9 \%)$ compared to others. $29.1 \%$ house wives were positive for IPI. Prevalence of IPI among students, farmers, house wives, merchants and governmental employees was 41.9\%(70/167), 60.3\%(38/63), $29.1 \%(14 / 48), 16.7 \%(6 / 36)$, and $9 \%(3 / 33)$, respectively. The association between occupation and IPI was statistically significant $(\mathrm{P}=0.006)$. From the total study subjects, 34(9.2\%), $101(27.4 \%), 29(7.9 \%), 31(8.5 \%)$ study subjects had monthly income of $<300$ birr, 300-900 birr, 1000-1500 birr and $>1500$ birr, respectively. Prevalence of IPI among study subjects who had monthly income of $<300$ birr was $45.4 \%$, 300-900 birr was $39.6 \%, 1000-1500$ birr was $17.2 \%$ and students, children and handicaps was $42 \%$. The monthly income and IPI was statistically associated $(\mathrm{P}<0.05)$. Out of the $174(47.3 \%)$ male and $194(52.7 \%)$ female study participants $67(38.5 \%)$ and $72(37.1 \%)$ were positive for at least one IP, respectively. However, there was no significant association between sex and IPI $(\mathrm{P}>0.05)$. Of 368 study subjects, $139(37.8 \%)$ were affected by IPI and 229(62.2\%) were not affected by IPI. From the total affected study participants, $50(36 \%), \quad 64(46 \%), \quad 13(9.4 \%)$ and $12(8.6 \%)$ study participants were in age groups $<14$ years, 15-29 years, 30-44 years and $>45$ years, respectively. Infection was more prevalent in the age groups of 15-29 years and $<14$ years compared to the other age groups and age was statistically associated with IPI $(\mathrm{P}=0.008)($ Table-2).

(Table-3).

Table 2. Distribution of IPs by sex and age group among patients requested for stool examination at Red Cross Clinic and Chelaleki Health Center April, 2014.

\begin{tabular}{|c|c|c|c|c|c|c|c|c|}
\hline Parameter & & & Para & ion & & & $X^{2}$ & p-value \\
\hline & Positive & & Nega & & Total & & \multirow{6}{*}{0.076} & \multirow{5}{*}{0.783} \\
\hline Sex & No. & Percent & No. & Percent & No. & Percent & & \\
\hline Male & 67 & 38.5 & 107 & 61.5 & 174 & 47.3 & & \\
\hline female & 72 & 37.1 & 122 & 62.9 & 194 & 52.7 & & \\
\hline Total & 139 & 37.8 & 229 & 62.2 & 368 & 100 & & \\
\hline \multicolumn{7}{|l|}{ Age } & & \multirow{5}{*}{0.008} \\
\hline$<14$ & 50 & 50 & 50 & 50 & 100 & 27.2 & \multirow{4}{*}{11.715} & \\
\hline $15-29$ & 64 & 37 & 109 & 63 & 173 & 47 & & \\
\hline $30-44$ & 13 & 26 & 37 & 74 & 50 & 13.6 & & \\
\hline$>45$ & 12 & 26.7 & 33 & 73.3 & 45 & 12.2 & & \\
\hline
\end{tabular}

The respondents that had habit of walking on barefoot were $70(19.6 \%)$, among them $15(21.4 \%)$ were affected by Hookworm and 55(78.6\%) were not affected by Hookworm. The respondents that had no habit of walking on bare foot were 298(80.4\%), among them 2(0.7\%) were affected by Hookworm and 296(99.3\%) not affected by Hookworm. Therefore, the relation between walking on barefoot and Hookworm infection was statistically significant $(\mathrm{P}<0.05)$. From the total respondents, 206(56\%) respondents had habit of hand washing before meal, among them, 77(37.4\%) were positive and $129(62.6 \%)$ were negative for IPI. $T$ he respondents that had no habit of hand washing before meal were $162(44 \%)$. Among them $62(38.3 \%)$ were positive and
$100(61.7 \%)$ were negative for IPI. The habit of hand washing before meal was not statistically associated with parasitic infection $(\mathrm{P}>0.05)$. Regarding habit of hand washing after defecation, $48.6 \%$ ( 179/368) had habit of hand washing after defecation. Among them 48(10.1\%) were positive and $131(89.9 \%)$ were negative for IPI. Whereas the respondents that had no habit of hand washing after defecation were $189(51.4 \%)$, among them $91(51.9 \%)$ were positive and 98(48.1\%) were negative for IPI. Therefore, the highest parasitic infection rate was observed in patients who had no habit of hand washing after latrine usage (defecation) and it was statistically associated with $\operatorname{IPI}(\mathrm{P}<0.05)$. Of 368 study subjects, 235(63.8\%) had trimmed finger nail, among them 
$85(36.2 \%)$ were positive for IPI. Hence, finger nail status was not statistically associated with IPI $(\mathrm{P}>0.05)$. Raw/undercooked meat and all of them were negative for
Taenea species. Habit of eating raw/undercooked meat were not statistically associated with IPI $(\mathrm{P}>0.05)$ (Table.3).

Table 3. Associations of independent variables with intestinal parasites at Red Cross Clinic and Chelaleki Health Center, East Wollega Zone, Ethiopia, April, 2014.

\begin{tabular}{|c|c|c|c|c|c|c|c|c|}
\hline \multicolumn{3}{|l|}{ Parameter } & \multicolumn{4}{|c|}{ Result of stool examination } & \multirow[t]{3}{*}{$\mathbf{X}^{2}$} & \multirow[t]{3}{*}{ p-value } \\
\hline & Positive & & Negative & & Total & & & \\
\hline Sex & No. & Percent & No. & Percent & No. & Percent & & \\
\hline Habits of walking on barefoot & 17 & 4.6 & 351 & 95.4 & 368 & 100 & 55.431 & 0 \\
\hline Habits of hand washing before meal & 139 & 37.8 & 229 & 62.2 & 368 & 100 & 0.031 & 0.860 \\
\hline Habits of hand washing after latrine use & 139 & 37.8 & 229 & 62.2 & 368 & 100 & 10.80 & 0.001 \\
\hline Finger nail status(trimmed or not) & 139 & 37.8 & 229 & 62.2 & 368 & 100 & 0.710 & 0.399 \\
\hline Habits of eating uncooked vegetables & 122 & 33.2 & 246 & 66.8 & 368 & 100 & 0.690 & 0.406 \\
\hline
\end{tabular}

\section{Discussion}

The demonstration that there was relatively high prevalence of IPI in this study was inconsistent with studies conducted in other countries $(9,24)$. For instance, studies conducted in Thailand, Sai-Yok District (28).Showed lower prevalence $(10.3 \% \%)$ of IPI than in the present study. Furthermore, lower prevalence $(6.2 \%)$ of IPI was reported from a study conducted among patients of Al-Noor specialist hospital in Saudi Arabia (9). In contrast, higher prevalence of IPI was reported from Southern Ethiopia (85.1\%)[35], Northern Gondar(79.8\%)(28)., Eastern Wollega(64.9\%)[36] and Teda Health Center, Northwestern Ethiopia(62.3\%)(17). The contradictory report on the prevalence of IPI could be due to variation in awareness regarding transmission and prevention of IPs between study participants in this study and previous studies. In addition, this relatively high overall prevalence of IPs might be due to poor environmental and personal hygiene, absence of adequate and safe water supply, habit of walking on bare foot, lack of education which increases overall magnitude of IPs and poor socio-economic living standards in our study area. In the present study, the prevalence of E. histolytica was reported to be $16.3 \%$ which is similar to a finding from a study conducted in Latin America (16.1\%) (29). In contrast, a study conducted among patients of Al-Noor specialist Hospital, Saudi Arabia (9). Showed lower prevalence of E. histolytica (4.7\%) (18). Furthermore, lower prevalence $(0.4 \%)$ of E. histolytica was reported from a study conducted in Malaysia (28). In Ethiopia, a study conducted in Delgi, Northern Gondar [34] showed higher prevalence of E. histolytica (27.3\%) than the present study. In contrast, lower prevalence of E. histolytica were reported from Southern Ethiopia (2.1\%) (25) and Northwestern Ethiopia (4.6\%)[9].The demonstration that there was $9 \%, 5.2 \%, 3.8 \%$ and $0.1 \%$ prevalence of G. lamblia, A. lumbricoides, Hookworm and $H$. nana was not in agreement with previous studies conducted in Northern Gondar [34] and Southern Ethiopia (25). For instance, as compared to the present study higher prevalence of $\mathrm{G}$. lamblia (41.6\%), Hookworm (48\%) and H. nana (11.5\%) were reported among Delgi school children, Northern Gondar (26). In contrast with this study,the prevalence of hookwarm,
S.mansoni and A.Lumbricoides were significantly higher in the age groups below 14 years and T.Trichiura in school children was much higher $21.08 \%$ than in the adult population $3.33 \%(31)$ In addition, a study conducted in Wondogenet, Southern Ethiopia (25). showed high prevalence of A. lumbricoides(25.7\%), G. lamblia(13.2\%), Hookworm $(5.9 \%)$ and H. nana $(4.5 \%)$. The present study did not find a significant difference between IPI and hand washing practice before meal $([\mathrm{P}>0.05)$. This was in agreement with a study conducted in Delgi, Northern Gondar (27).Furthermore, a significant difference was observed in hand washing practice after defecation with IPI. Similar finding was reported from a study conducted in Teda Health Center, Northwestern Ethiopia (9). The contrary association between hand washing practice after defecation and IPI might be due to the habit of using only water for washing in the area and inappropriate handling of readymade foods and drinks, without washing their hands using soap/ash. The present study also found that using water from a river and unprotected well/spring were risk factors for intestinal parasitic infection. This may arise from the contamination of water with animals and human waste that flooding in to the river or unprotected spring. However, other unknown factors may contribute to the increased risk associated with river/spring water and merit further investigation .Habit of walking on barefoot and less shoes wearing habits showed a statistically significant association with Hookworm infection. This result was consistent with a study conducted in Northwestern Ethiopia $(27,28,30)$. This might be due to lack of awareness of patients on how Hookworm is transmitted to human and walking on bare foot instead of wearing shoes allowing penetration of skin by infective filariform larvae. However, sex, religion, habit of trimming finger nail, habit of eating uncooked vegetable, and habit of eating raw/undercooked meat did not show statistically significant associations with IPIs.

\section{Strength and Weakness}

\subsection{Strength of the Study}

The data was collected using a structured self-administered pre-tested questionnaire 
Adequate sample size was applied according to single population proportion formula

Data collators were health professional

\subsection{Weakness of the Study}

During this study there may be observational bias

There was financial constraint while conducting the Study

All respondents were interviewed but some of them observed during the study period

Some of the respondents were volunteer during data collection

\section{Conclusion}

In general, the present study showed high prevalence of IPs in the study area. Furthermore, age, absence of hand washing habit after defecation, occupation and income status were found to be associated with IPI. Source of drinking water supply and habit of walking on bare foot showed a statistically significant association with prevalent E. histolytica and Hookworm infections, respectively. There is a need for integrated control programs including periodic de-worming, creating awareness regarding the importance of washing hands after defecation and impact of using contaminated water to have a lasting impact on transmission of IPs.

\section{Acknowledgement}

It is hereby acknowledged colleagues and all those who associated in conducting the study or critiquing the manuscript.

\section{Author's Contribution}

Development of the original idea and protocol, data abstraction and analyses, writing the manuscript: Addis Adera Gebru, Birhan Alemnew, Yonas Yimam Ayenem and Tefera Nigussie: and Development of the protocol, over all Guide data abstraction, preparing the manuscript: Zemenu Mengistie Semene, Ambachew Woreta Hailu, Markos Kidane Assefa.

\section{Funding /Support}

The study was not supported by any grant and was part of interested Authors.

\section{References}

[1] Heclan, J., Ingerson, F (2002). Essential human parasitological. India; 1:4-9.

[2] Carneiro, F., Cifuentes, E., M. Tellez-Rojo, M (2000). "Environmental health indicators and development of preventive actions against Ascaris lumbricoides infections in rural communities, Caparao and Alto Caparao, Minas Gerais, Brazil" Epidemiology; 11(4):32-45.

[3] Fincham, JE, Markus, MB. , Adams, VJ (2003). Could control of soil-transmitted helimnthic infection influence the HIV/AIDS pandemic. Acta Trop 86:315-333.

[4] De Silva, NR, Brooker, S., Hotez, PJ, Montresor A, Engels D, et al (2003) Soil-transmitted helminth infections: Updating the global picture. Trends parasitol 19:547-551.

[5] Same A, Guerrant, R L, Barre, HL, Bessong, PO, Iqumbor, EO, Obi, Ch. (2009). Prevalence of intestinal parasitic and bacterial pathogens in Diarrheal and non-diarrheal human stools from Vhembe District, South Africa. Journal of health, population and nutrition $\mathrm{pp} 739-745$

[6] Adams VJ, Markus MB, Adams JFA, Jordan E, Curtis B, Dhansay MA, et al.(2005 ) paradoxical helminthiasis and giardiasis in Cape town, south Africa: epidemiology and control. African health sciences 5, pp, 276-280

[7] Chandrasekhar, MR., Nagesha, CN. (2003). Intestinal helimnthic infestation in children. Indian journal of pathology and microbiology 46,pp 492-494

[8] Cook DM, Swanson RC, Eggett, DL, Booh GM. (2009). A retrospective analysis of prevalence of gastro intestinal parasites among school children in the Palajunoj valley of Guatemala. Journal of health, population and nutrition.

[9] Zaglool DAM, Khodari YAW, Gazzaz ZJ, Dhafar KO, Shaker HAS, Farook MV. (2011). Prevalence of intestinal parasites among patients of Al-Noor specialist Hospital, Makkah, Saudi Arabia. Oman Med J.; 26(3):182-185.

[10] Federal Democratic Republic of Ethiopia (FDRE) (1998). Health and health related indicators. Health information processing and documentation team, planning and project department. Ministry of Health, Ethiopia. 35 pages

[11] Ministry of Health (MOH) (2004) Health and health related indicators. Planning and programming department, FMOH, Addis Ababa, Ethiopia.

[12] Roma, B.,Worku,S (1997). Magnitude of schistosoma mansoni and intestinal helimnthic infections among school children in Wondogenet Zuria, Southern Ethiopia Ethiop J Health Dev 11:125-129.

[13] Kumie, A, Ali A (2005). An overview of environmental health status in Ethiopia with particular emphasis to it is organization, drinking water and sanitation: A literature survey. Ethiop J Health Dev 2005; 19(2):89-103.

[14] Cleave land S, Laurenson, MK., Tayler, LH (2001). Diseases of humans and their domestic mammals: pathogen characteristics, host range and the risk of emergency. Phil Trans R sac land B 2001; 356:991-999

[15] Wolde Michael, T., Endeshaw, T., Sshibre, T., Gebre, T., Haddis, M., Tilahun, D, et al (1999). Intestinal parasitic infections in Western Abaya with special reference to schistosoma mansoni. Ethiop J Health Dev 13:25-26.

[16] Alemu, M., Hailu, A., Buggsa, G(2014). Prevalence of intestinal Schistosomiasis and soil transmitted heliminthiasis among primary school children in umolante district, South Ethiopia. Clinical Medicine Research. 3960: 174180.doi.10.11648/j.cmr.20140306.14.http://www.sciencepubli shinggroup.com $/ \mathrm{j} / \mathrm{cmr}$.

[17] WHO, 2004. World health report 2004: changing history. Geneva World organization 
[18] Abraraw A, Biniam K, Eylachew B, et al (2013).Cross sectional study on the prevalence of intestinal parasites and associated risk factors in Teda Health Center, Northwest Ethiopia. ISRN Parasitology, 2013; pp1-5

[19] Mahfouz, AAR, EI-Morishedy, H., Farghaly, A.,Khalil, A (1997).Ecological determinants of intestinal parasitic infections among preschool children in an urban squatter settlement of Egypt. J Trop pediatric 1997; 43:341-344

[20] Phiri, K., Whitty, CJ, Graham, SM., Sembatya, S., Lule, $\mathrm{G}(2000)$ :.Urban /Rural distance in prevalence of intestinal helminthes in southern Malawi. Ann Top med parasite. 2000 ; 94:381-387

[21] Cheesbrough, M (1998). District Laboratory Practice in Tropical Countries. Part one.

[22] Bethony J, Brooker S, Albonico M, Geiger SM, Loukas A, et al. (2006) Soil-transmitted helimnthic infections : Ascariasis, Trichuriasis, and Hookworm. Lancet 367:1521-1532.

[23] Jemaneh, L (2000). The epidemiology of schistosomamansoni and soil-transmitted helminthes in elementary school children from the south Gondar zone of the Amhara National Regional state, Ethiopia. Ethiop Med J 38:105-118.

[24] Teera, K., Wamma, M., Ssupaporn, N., Wichit, R, A., Morn ,L.(2011). Parasitic and bacterial infections among food handlers in kanchanaburi, Thailand. The Journal of Tropical medicine and Parasitology. 2011; 34:49-53.

[25] Liza, A., Nyantekyi., Mengstu L., Mulugeta, B,Konjit, T., Kebreten, M., Chanda ,M(2010). Intestinal parasitic infections among under-five children and maternal awareness about the infections in SheshaKekele, Wondo Genet, Southern Ethiopia. Ethiop J Health Dev; 24(3):185-190.

[26] Asrat, A., Tewodros, D., Alemayehu, W (2011). Prevalence and risk factors of IPs among Delgi school children, Northern Gonder, Ethiopia. Journal of Parasitology and Vectoriology. 2011; 3(5), 75-81.

[27] Dori, Gu., Tullu, KD, Ali, L., Hirko, A., Mekuria ,G(2011). prevalence of hook worm infection and its association with anemia among patients visiting Fenan Medical Center, East Wollega , Ethiop Med J. 2011; 49(3):265-271.

[28] Jamaiah, I., Rohela, N (2005).. Prevalence of IPs among members of the public in Kuala Lumpur, Malaysia SE Asian J Trop Med. Public Health. 36 (1): 68-70.

[29] Anjum, M (2007). Hook worm infection and its correlation with packed cell volume in a rural community of Pakistan. Professional Med J.14:33-35

[30] Chanyalew, MD., Gurara, MK(2014). Malaria and Intestinal parasite infections and co-infections in Tach Gayint District, South Gondar Zone, Amhara Regional State 2010. Science Journal of Public Health.296):546553.doi.10.11648/j.sjph.20140206.18.http://www.sciencepubli shinggroup.com/j/sjph.

[31] Wang, LD., Guo, JG, WU, XH., Wang ,TP.., Zhusp, Zhang, ZH, Stainmanni, P., Yang, GJ., Wang ,SP, et al(2009). China's new strategy to block schistosomajaponicum transmission experiences and impact beyond schistosomeasis. Trop Med Int Health.; 14:475-483 